\title{
Manipulables físicos para la formación de conceptos artificiales en niños de 6 a 8 años de $\epsilon$ dad
}

\begin{abstract}
Luis Alejandro Andrade Lotero*, María Fernanda Cobo Charry**, Lizeth Natali Díaz Díaz, Andrea Juliana Flórez Pineda, Constanza Garavito Muñoz, Diana Patricia González Doblado, Edit Alexandra Hernández Rojas, Sandra Milena Parra Rojas, Guiomar Adrea Villarraga Acero***
\end{abstract}

Resumen. Esta publicación hace parte de una investigación más general, la cual busca estudiar los procesos de aprendizaje con manipulativos en tres diferentes sustratos: físico, digital-mouse y digital-pantalla-táctil. El presente texto

* Director de trabajo de grado. Antropólogo, Magíster en Educación. Investigador Asociado Fundación Alandra-DifuCiencia. Integrante del grupo de investigación Tendencias Actuales en Pedagogía y Educación. Línea de investigación. formación y práctica pedagógica. al.andrade@ alandradifuciencia.org

** Codirectora del trabajo de grado. Psicóloga. Magíster en Educación. Profesora Facultad de Educación, Universidad de San Buenaventura, sede Bogotá. Integrante del grupo de investigación Tendencias Actuales en Pedagogía y Educación. Línea de investigación. formación y práctica pedagógica.macobo@academia.usbbog.edu.co

*** Estudiantes que optan por el título de Licenciadas en Educación para la Primera Infancia, Universidad de San Buenaventura, sede Bogotá. 
corresponde a la fase de manipulativos físicos para estudiar la formación de conceptos artificiales. La presente investigación tuvo un enfoque cualitativo de tipo descriptivo, y realizó una adaptación, diseño y pilotaje del Método de la doble estimulación originalmente trabajado por Vigotsky. La muestra consistió en 7 niños y niñas de 6 a 8 años de edad. Durante el análisis de resultados se evidenció que el desarrollo de conceptos no es homogéneo de acuerdo a la edad. Además, se encontró que los sujetos de 8 años son más minuciosos y observadores, por lo que toman más tiempo para clasificar los objetos. Adicionalmente, la mayoría de participantes en la prueba piloto presentaron dificultad al momento de expresar verbalmente la palabra oculta a la cuál pertenecían los objetos. Para finalizar, es conveniente decir que los manipulativos físicos son esenciales en las edades entre los 6 y 8 años, ya que brindan al niño la oportunidad de tocar, coger, mover, trasladar y organizar su material de aprendizaje. De esta manera, se les facilita observar las características de los objetos y los detalles de los mismos, con el fin de formar un concepto potencial.

Palabras claves. Aprendizaje; Constructivismo; Desarrollo Cognoscitivo; Formación de Conceptos; Manipulativos.

Abstract. This paper is part of a more general research, which aims to study the learning process with manipulatives on three different substrata: physical, digital-mouse, and digital-touch-screens. This document corresponds to the physical-manipulatives phase, with which we intend to explore the formation of artificial concepts. This research employed a qualitative-descriptive method, and adapted, designed and piloted the 'Double Stimulation Method', originally developed by Vygotski. The participants were 
seven 6-8 year old children. The data analysis showed that the development of concepts is not a homogeneous process with respect to age. Additionally, we found that 8 year old subjects were more meticulous observers, and took more time to classify objects. Furthermore, most of the participants had difficulties when asked to verbally express the hidden word attributes. Finally, we illustrate how physical manipulatives are important for children at these ages. Essentially, they need to touch, take, move and organize the learning materials. In such a way, children find it easier to observe the details and characteristics of the objects to form a new concept.

Keywords. Concept Formation; Cognitive Development; Constructivism; Learning; Manipulatives.

\section{Introducción}

Anteriores investigaciones han señalado la importancia de las acciones físicas para apoyar procesos de alto nivel cognitivo (Martin \& Schwartz, 2005). En este sentido, Manches y O'Malley (en prensa) exponen la necesidad de evaluar cómo la acción con manipulativos conduce al aprendizaje. Estos autores proponen valorar las ventajas de los manipulativos desde dos perspectivas: por un lado, la descarga cognitiva; y por el otro, la función metafórica conceptual (Manches \& O'Malley, en prensa). Esto quiere decir, por una parte, que los manipulativos apoyan la resolución de tareas descargando procesos cognitivos al entorno (Ainsworth, 2006; Artino, 2008; Orgill \& Crippen, 2010). Y por otra, que éstos apoyan el "mapeo" entre un conocimiento de dominio tangible y material, hacia un conocimiento de dominio conceptual (Ainsworth, 2006; Núñez, Edwards, \& Matos, 1999). 
Por su parte, la formación de conceptos ha sido un tema que se ha investigado anteriormente. Algunos de estos estudios se han llevado a cabo por medio de experimentos, dentro de los cuales cabe destacar el "Método de la Doble Estimulación" propuesto originalmente por Vigotsky (1995). Esta técnica permitió observar y analizar la dinámica interna del proceso de formación de conceptos. En su estudio, Vigotsky describió cuatro niveles de formación de un concepto, a saber, cúmulos, complejos, pseudo-conceptos y conceptos potenciales. Del mismo modo, Luria (1995) planteó cuatro métodos para investigar el desarrollo de los conceptos. Luria mencionó la importancia de la palabra, qué sentido tiene la misma en cada etapa del desarrollo y cómo varía éste de acuerdo con la edad. Dentro de los métodos que Luria planteó se describe cómo los niños pueden clasificar, organizar y categorizar objetos según la edad y el medio en el que se encuentran. La presente investigación hizo uso de la prueba "Cuarto Excluido" y de formación de conceptos artificiales (Luria, 1995).

\section{Formación de conceptos desde el enfoque histórico cultural de Vigotsky}

Según Vigotsky, la formación de conceptos tiene como característica ser un proceso creativo, donde la actividad y la palabra son igual de importantes, ya que el lenguaje es un medio que luego se convierte en símbolo de concepto (Vigotsky, 1995). Vigotsky utilizó el experimento conocido como Método de la Doble Estimulación para estudiar los conceptos y su formación. Este experimento consistía en presentar al sujeto 22 trozos de madera de color, forma, peso, y tamaño variados. Los trozos de madera presentaban 5 colores diferentes, 5 formas diferentes, 2 alturas (altos y chatos) y dos tamaños de la superficie horizontal (grande y pequeño). Las 22 piezas tenían en un costado oculto una palabra sin sentido. Cada palabra estaba asociada con dos atributos en común (ver figura 2). 


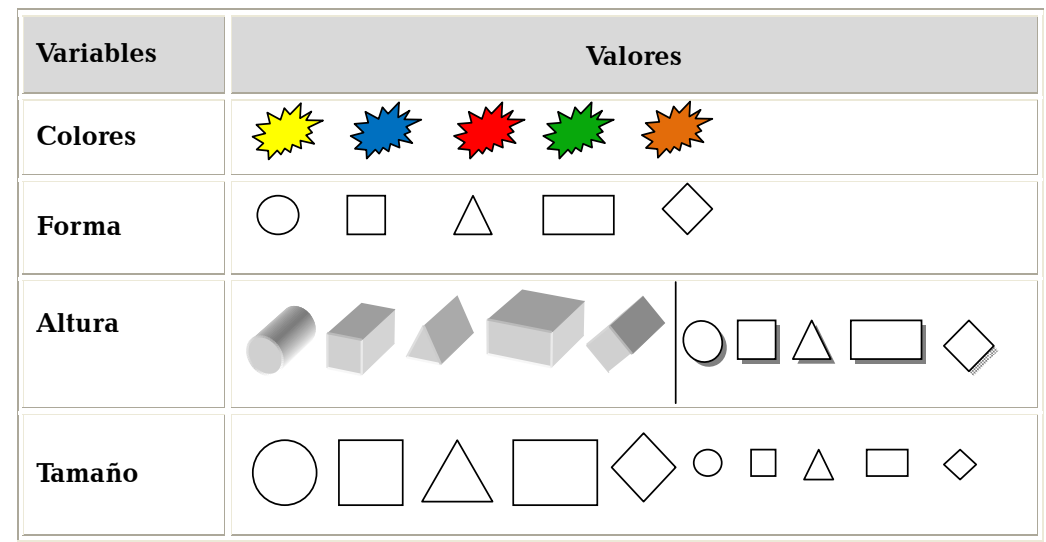

Figura 2. Experimento original de Vigotsky "Método de la Doble Estimulación" (Vygotsky, 1995).

Según Vigotsky, el concepto se forma de una operación intelectual en la cual las funciones mentales elementales participan en una combinación específica, dirigida principalmente por el uso de la palabra para abstraer o sintetizar ciertos rasgos y posteriormente ser simbolizados por el sujeto. Vigotsky demostró que la existencia de asociaciones, aunque numerosas y fuertes, entre los símbolos verbales y los objetos no es en sí misma suficiente para la formación de conceptos. El uso de la palabra es una parte integral del proceso de desarrollo, cuya función es direccionar la formación de conceptos genuinos, a los que conducen estos procesos (Vygotsky, 1995).

\section{I.I. Niveles de formación de conceptos}

La formación de un concepto es el resultado de una actividad compleja en la cual intervienen las funciones intelectuales básicas y superiores (Vigotsky, 1995). Vigotsky divide el proceso de formación de conceptos en cuatro etapas, a saber: cúmulos inorganizados, pensamiento en complejos, pseudo-conceptos y conceptos potenciales. A continuación se explicarán cada una de las cuatro etapas: 


\section{I.I.I. Cúmulos Inorganizados}

Es cuando los sujetos ubican juntos un número de objetos, los cuales son agrupados sin ningún fundamento. En esta etapa, la palabra no es significante para el niño, ya que ellos asocian los objetos por lo que un adulto puede describirles (Vigotsky, 1995). Los cúmulos inorganizados se dividen en tres etapas: La primera es el agrupamiento, aquí se ve el ensayo y error en el desarrollo del pensamiento, es creado al azar y no tiene fundamento de algo en específico. La segunda es una organización del campo visual, se debe a la posición espacial de los objetos. La tercera es la imagen acumulada, que se basa en algo más complejo, pero que sigue teniendo incoherencia en la formación de grupos.

\section{I.I.2. Pensamiento en Complejos}

En esta fase los objetos individuales se unen en la percepción del niño a través de vínculos coherentes y verdaderos. Según Vigotsky, el pensamiento en complejos es ya un pensamiento vinculado y objetivo, aunque no refleja las relaciones objetivas del mismo modo que el pensamiento conceptual. Dentro de los complejos se encuentran: los asociativos, colecciones, complejos en cadena, amalgama psíquica y complejo difuso (Vigotsky, 1995).

\section{I.I.3. Pseudo-conceptos}

Se da cuando el niño encierra un ejemplo con objetos que hubiera podido agrupar basándose en un contenido indefinido. Estos se manifiestan de manera clara cuando el niño se guía de una semejanza puntual y visible. Esta fase es indispensable en la formación de conceptos ya que es un puente entre el pensamiento en complejos y la verdadera formación del concepto, puesto que el primero es aquel que porta las bases suficientes para este último.

\section{I.I.4. Conceptos potenciales}

En esta última fase, al ser humano se le hace necesario abstraer y separar los elementos y considerarlos aparte de la totalidad de la 
experiencia concreta en la cual están encajados. La abstracción se da cuando el niño agrupa en un mismo conjunto los objetos que tienen mayor semejanza, siendo todos los objetos distintos. Posteriormente, el niño pasa a agrupar objetos teniendo en cuenta un atributo en común y es allí donde surgen los conceptos potenciales.

\section{I.2. Conceptos científicos y conceptos de la vida cotidiana}

Vigotsky definió los conceptos cotidianos como aquéllos que se encuentran o desarrollan en un momento particular y específico, y denominó conceptos científicos a aquéllos que desarrolla el sujeto introduciendo a un objeto dado dentro de en un sistema de determinaciones lógico-verbales (Luria, 1995). Los conceptos científicos se encuentran dentro de un desarrollo espontáneo provocado por la acción pedagógica. Por tal razón, se da en el proceso de enseñanza o en contextos específicos. Según Vigotsky, la construcción de conceptos científicos inicia con la definición verbal para transformarla en concepto (Baquero, 1996).

Específicamente, en la adaptación que se realizó del Método de la Doble Estimulación se trabajaron los conceptos cotidianos como el color, la forma, el tamaño, y otros caracteres visibles. El experimento partió de conceptos cotidianos que los niños ya conocían y se encontraban continuamente con ellos en su diario vivir.

\section{Metodología}

La presente investigación tuvo un enfoque cualitativo de tipo descriptivo. Asimismo, se utilizaron técnicas cualitativas en la obtención y análisis de los datos (Hernández, Fernández, \& Baptista, 2008). La investigación se abordó como una adaptación, diseño y pilotaje del método de la doble estimulación (Vigotsky, 1995). La muestra consistió en 7 niños y niñas de 6 a 8 años de edad en un colegio a las afueras de la ciudad. Los datos se obtuvieron utilizando la observación directa, el registro en video y en diarios de 
campo. La investigación siguió un diseño aplicando un pre-test, seguido del pilotaje de la prueba de intervención y, finalmente, la aplicación de un post-test. El análisis cualitativo de los datos se realizó a través de un estudio de contenido de las tres pruebas aplicadas.

A continuación se describen las diferentes fases del diseño de la investigación.

\section{I Descripción de los manipulativos físicos}

Se propusieron tres tipos de objetos para que sirvieran de manipulativos físicos, a saber, casas, muñecas y carros (ver anexo 1).

2.1.1. Los atributos a observar en las casas fueron los techos, ventanas y nivel de piso. Los techos se diferenciaron por ser de paja, de tejas y de fomy. En las ventanas se tuvieron en cuenta dos atributos, basados en forma circular y cuadrada. En los niveles de piso se observó el número de pisos que tenían, que podían ser de uno o dos pisos (ver figura 3.).

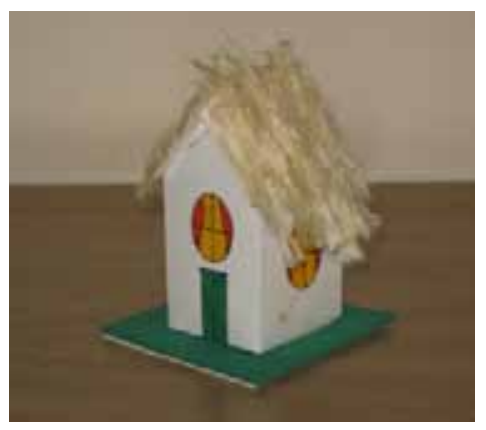

Figura 3. Atributo de Casas.

2.1.2. Los atributos a observar en las muñecas fueron tres: bolso, oso, y peinado -este último podía ser cola de caballo, cebollitas o dos trenzas (ver figura 4.). 


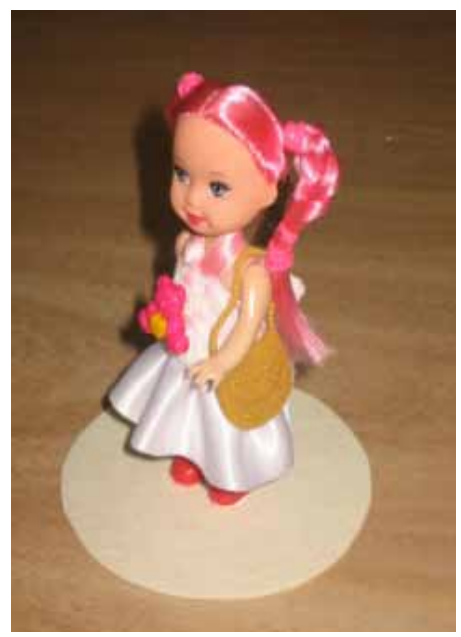

Figura 4. Atributo de Muñecas.

2.1.3. Los atributos que caracterizaron a los carros (ver figura 5.) fueron los autoadhesivos, la llanta trasera, y el color (naranja, rojo y morado).

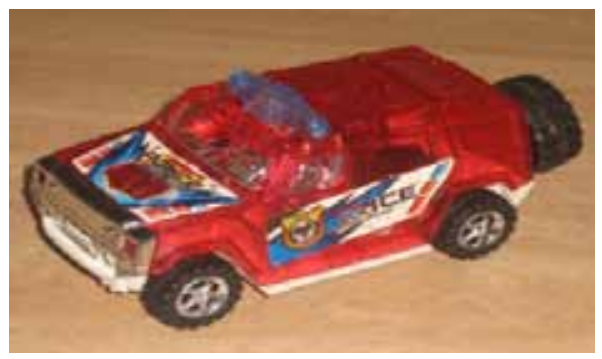

Figura 5. Atributo de Carros.

\subsection{Diseño del protocolo de intervención}

Los protocolos de intervención fueron adaptados para cada uno de los objetos diseñados, teniendo en cuenta los distintos atributos y palabras ocultas. El protocolo de instrucción se 
elaboró con el fin de describir paso a paso cómo debe ser aplicada la prueba adaptada del método de la doble estimulación. El esquema principal concebido fue iniciar saludando al sujeto y seguido dar la instrucción del juego. Posteriormente, se da al sujeto un ejemplo de la palabra oculta. Si éste no lograba realizar la clasificación del atributo determinado, se le ofrecía nuevamente otro ejemplo. Si aún así el sujeto no lograba clasificar adecuadamente el material, en el tercer intento se le presentaba al sujeto un contraejemplo. Estos pasos se repetían hasta asegurarse que el sujeto descubriera el significado de la palabra artificial.

\subsection{Diseño del Pre-test}

En primer lugar, se le presentó al sujeto un juego de tarjetas del cuarto excluido (Luria, 1995). Este consistió en mostrar cuatro tarjetas con imágenes de objetos, tres relacionados entre sí, es decir, pertenecientes a una categoría, y una de ellas que no pertenecía a dicha categoría. En esta investigación se utilizaron tres juegos de tarjetas con diferentes objetos (ver anexo 2). Se informó a los sujetos que deberían descartar el objeto que no pertenecía al grupo y sustentar el porqué dicha tarjeta no correspondía con las demás. Posteriormente, si el sujeto lograba separar las tres pruebas del cuarto excluido, se daba inicio a la prueba piloto del experimento adaptado "Método de la Doble Estimulación".

\subsection{Diseño de la intervención}

Se presentó a los sujetos los manipulativos físicos para la realización de la prueba piloto. Ésta se desarrollo de acuerdo a un protocolo de instrucción y no hubo un orden establecido para la presentación del material. La prueba consistió en presentar al sujeto un grupo de objetos que debía relacionar y agruparlos de acuerdo a una palabra sin sentido que hacía referencia a un atributo en común. 


\subsection{Diseño del Post-test}

A cada sujeto se le pidió que representara gráficamente lo qué él o ella recordaba del significado de cada una de las palabras sin sentido. Para ello, se le facilitó al sujeto una hoja en blanco y se le dejó un tiempo prudencial para que dibujara. En el proceso, un investigador acompañó al sujeto.

\section{Resultados}

\section{I. Resultados del pre-test}

En la tabla 2 se presentan las respuestas dadas por los niños en el pre-test. Estas se clasificaron en tres tipos de razones diferentes: categoría abstracta, situación práctica y rasgos concretos. La categoría abstracta muestra que más de la mitad de los sujetos coincidió en decir a qué grupo pertenecía cada conjunto de elementos (más de cuatro de siete niños en total). En la situación práctica, una minoría clasificó haciendo referencia a la utilidad que prestan estos elementos (aproximadamente dos de siete). Finalmente, en la categoría de rasgos concretos, sólo algunos sujetos clasificaron los objetos de acuerdo a las cualidades concretas de los mismos (entre dos y cuatro de siete). En conclusión, se deduce que los niños relacionan y clasifican objetos de diferentes maneras, usualmente incluyéndolos en una categoría determinada.

A su vez, en la tabla 3 se muestra, según la edad, qué razones emplearon los sujetos para clasificar los objetos. Las respuestas dadas por los niños se evidenciaron en tres categorías particulares: categoría abstracta, situación práctica y rasgos concretos. Finalmente, se considera que los niños de menos edad tienden a clasificar de acuerdo a cualidades visibles o relacionando los objetos con situaciones de la vida cotidiana, mientras que los de mayor edad, incluyen los objetos en categorías determinadas. 
Tabla 2. Razones que dieron los participantes en el pre-test.

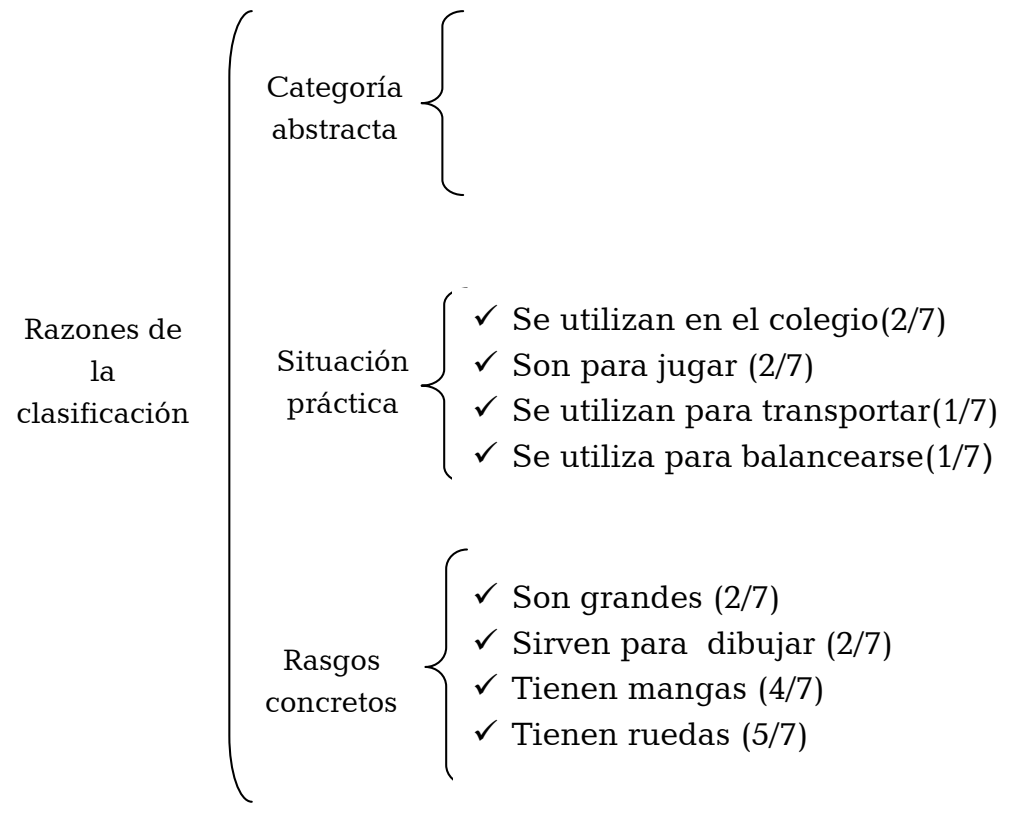

Tabla 3. Frecuencia de razones de categorización por edad.

\begin{tabular}{|c|c|c||c||}
\hline Edad & Categoría abstracta & Situación práctica & Rasgos Concretos \\
\hline \hline 6 & 0 & 1 & 1 \\
\hline \hline 7 & 0 & 2 & 0 \\
\hline \hline 8 & 2 & 1 & 0 \\
\hline
\end{tabular}

\subsection{Resultados de la prueba de intervención}

En la tabla 4 se muestra el número de sujetos que participó en el pilotaje de las pruebas realizadas con el material tangible y los protocolos que cada uno de ellos desarrolló. Los protocolos del 1 al 3 pertenecen al material de casas; del 4 al 6 al material de muñecas; y del 7 al 9 corresponden al material de carros. Aquí se observa que la mayoría de los niños participó en el desarrollo de 
tres protocolos sin importar el material o el número de protocolo a desarrollar. Finalmente, se puede observar que ningún material tuvo prioridad respecto a los otros.

Tabla 4. Protocolos realizados por los sujetos.

\begin{tabular}{|r||c||c|c||c|c|c||c|c|c||}
\hline \multirow{2}{*}{ Sujeto } & \multicolumn{3}{|c||}{ Protocolo casas } & \multicolumn{2}{c||}{ Protocolo muñecas } & \multicolumn{3}{|c|}{ Protocolo carros } \\
\cline { 2 - 10 } & 1 & 2 & 3 & 4 & 5 & 6 & 7 & 8 & 9 \\
\hline \hline 1 & & & & $\mathrm{X}$ & $\mathrm{X}$ & $\mathrm{X}$ & & & \\
\hline \hline 2 & & & & $\mathrm{X}$ & & & $\mathrm{X}$ & & \\
\hline \hline 3 & $\mathrm{X}$ & & & & $\mathrm{X}$ & & & $\mathrm{X}$ & \\
\hline \hline 4 & & & $\mathrm{X}$ & $\mathrm{X}$ & & & & $\mathrm{X}$ & \\
\hline \hline 5 & $\mathrm{X}$ & $\mathrm{X}$ & $\mathrm{X}$ & & & & & & \\
\hline \hline 6 & & & & & & & $\mathrm{X}$ & $\mathrm{X}$ & $\mathrm{X}$ \\
\hline \hline 7 & & & & $\mathrm{X}$ & $\mathrm{X}$ & $\mathrm{X}$ & & & \\
\hline
\end{tabular}

A continuación en una serie de tablas, se presentan los protocolos que cada sujeto desarrolló frente al número de ayudas y el tiempo que necesitó para descubrir la palabra oculta en cada material.

Tabla 5.1. Ayudas utilizadas y tiempo de duración por el sujeto 1.

\begin{tabular}{|c||c||c||c||}
\hline $\begin{array}{c}\text { Nombre del } \\
\text { protocolo aplicado }\end{array}$ & $\begin{array}{c}\text { N. }{ }^{\circ} \text { del protocolo } \\
\text { aplicado }\end{array}$ & $\begin{array}{c}\text { N. }{ }^{\circ} \text { de ayudas } \\
\text { utilizadas }\end{array}$ & $\begin{array}{c}\text { Tiempo de } \\
\text { duración }\end{array}$ \\
\hline \hline muñecas & 4 & 3 & $10 . \mathrm{min}, 51 \mathrm{seg}$ \\
\hline \hline muñecas & 5 & 3 & $6 \mathrm{~min}, 39 \mathrm{seg}$ \\
\hline \hline muñecas & 6 & 2 & $3 \mathrm{~min}, 58 \mathrm{seg}$ \\
\hline
\end{tabular}

En la tabla 5.1., se observa que el sujeto 1 desarrolló los tres protocolos de un mismo material. Se puede apreciar que en el último protocolo que desarrolló necesitó menos ayudas que los anteriores. Con respecto al tiempo, se muestra que mejoró a medida que avanzaba en cada juego. 
Tabla 5.2. Ayudas utilizadas y tiempo de duración por el sujeto 2 .

\begin{tabular}{|c||c||c||c||}
\hline $\begin{array}{c}\text { Nombre del } \\
\text { protocolo aplicado }\end{array}$ & $\begin{array}{c}\text { N. }{ }^{\circ} \text { del protocolo } \\
\text { aplicado }\end{array}$ & $\begin{array}{c}\text { N. }{ }^{\circ} \text { de ayudas } \\
\text { utilizadas }\end{array}$ & $\begin{array}{c}\text { Tiempo de } \\
\text { duración }\end{array}$ \\
\hline \hline muñecas & 4 & 2 & $14 \mathrm{~min}, 46 \mathrm{seg}$ \\
\hline \hline carros & 7 & 2 & $1 \mathrm{~min}, 9 \mathrm{seg}$ \\
\hline
\end{tabular}

En la tabla 5.2., se observa que el sujeto 2 realizó sólo dos protocolos y con dos materiales diferentes. Para cada material necesitó el mismo número de ayudas. En el primer protocolo duró mucho más tiempo en descubrir la palabra oculta que en el segundo.

Tabla 5.3. Ayudas utilizadas y tiempo de duración por el sujeto 3 .

\begin{tabular}{|c||c||c||c||}
\hline $\begin{array}{c}\text { Nombre del } \\
\text { protocolo aplicado }\end{array}$ & $\begin{array}{c}\text { N. }{ }^{\circ} \text { del protocolo } \\
\text { aplicado }\end{array}$ & $\begin{array}{c}\text { N. }{ }^{\circ} \text { de ayudas } \\
\text { utilizadas }\end{array}$ & $\begin{array}{c}\text { Tiempo de } \\
\text { duración }\end{array}$ \\
\hline \hline casas & 1 & 2 & $3 \mathrm{~min}, 58$ seg \\
\hline \hline muñecas & 5 & 1 & $2 \mathrm{~min}, 21 \mathrm{seg}$ \\
\hline \hline carros & 8 & 1 & $1 \mathrm{~min}, 33 \mathrm{seg}$ \\
\hline
\end{tabular}

En la tabla 5.3., se observa que el sujeto realizó un protocolo de cada material; para el primero necesitó dos ayudas, para los dos siguientes sólo uno. Los protocolos los realizó de forma rápida; éstos no pasaron de 5 minutos.

Tabla 5.4. Ayudas utilizadas y tiempo de duración por el sujeto 4 .

\begin{tabular}{|c||c||c||c||}
\hline $\begin{array}{c}\text { Nombre del } \\
\text { protocolo aplicado }\end{array}$ & $\begin{array}{c}\text { N. }{ }^{\circ} \text { del protocolo } \\
\text { aplicado }\end{array}$ & $\begin{array}{c}\text { N. }{ }^{\circ} \text { de ayudas } \\
\text { utilizadas }\end{array}$ & $\begin{array}{c}\text { Tiempo de } \\
\text { duración }\end{array}$ \\
\hline \hline casas & 3 & 2 & $3 \mathrm{~min}, 5 \mathrm{seg}$ \\
\hline \hline muñecas & 4 & 1 & $2 \mathrm{~min}, 48 \mathrm{seg}$ \\
\hline \hline carros & 8 & 1 & $2 \mathrm{~min}, 25 \mathrm{seg}$ \\
\hline
\end{tabular}

En la tabla 5.4., se observa que el sujeto realizó tres protocolos, uno por cada material. Al igual que el sujeto anterior, utilizó el mínimo de ayudas para descubrir la palabra oculta y no pasó de los 5 minutos de tiempo. 
Tabla 5.5. Ayudas utilizadas y tiempo de duración por el sujeto 5 .

\begin{tabular}{|c||c||c||c||}
\hline $\begin{array}{c}\text { Nombre del } \\
\text { protocolo aplicado }\end{array}$ & $\begin{array}{c}\text { N. }{ }^{\circ} \text { del protocolo } \\
\text { aplicado }\end{array}$ & $\begin{array}{c}\text { N. }{ }^{\circ} \text { de ayudas } \\
\text { utilizadas }\end{array}$ & $\begin{array}{c}\text { Tiempo de } \\
\text { duración }\end{array}$ \\
\hline \hline casas & 1 & 2 & $3 \mathrm{~min}$ \\
\hline \hline casas & 2 & 3 & $13 \mathrm{~min}, 29 \mathrm{seg}$ \\
\hline \hline casas & 3 & 5 & $18 \mathrm{~min}$ \\
\hline
\end{tabular}

En la tabla 5.5., se observa que el sujeto 5 desarrolló tres protocolos de un mismo material. Se puede apreciar que inició utilizando el mínimo de ayudas, pero al avanzar con los otros protocolos presentó mayor dificultad y aumentó el número de ayudas así como también aumentó el tiempo para descubrir la palabra oculta.

Tabla 5.6. Ayudas utilizadas y tiempo de duración por el sujeto 6 .

\begin{tabular}{|c||c||c||c||}
\hline $\begin{array}{c}\text { Nombre del } \\
\text { protocolo aplicado }\end{array}$ & $\begin{array}{c}\text { N. }{ }^{\circ} \text { del protocolo } \\
\text { aplicado }\end{array}$ & $\begin{array}{c}\text { N. }{ }^{\circ} \text { de ayudas } \\
\text { utilizadas }\end{array}$ & $\begin{array}{c}\text { Tiempo de } \\
\text { duración }\end{array}$ \\
\hline \hline carros & 7 & 1 & $3 \mathrm{~min}$ \\
\hline \hline carros & 8 & 2 & $3 \mathrm{~min}, 36 \mathrm{seg}$ \\
\hline \hline carros & 9 & 3 & $9 \mathrm{~min}, 37 \mathrm{seg}$ \\
\hline
\end{tabular}

En la tabla 5.6., se observa que el sujeto 6 realizó tres protocolos de un mismo material. Se puede apreciar que aumentó el número de ayudas a medida que avanzó, pero, en general, utilizó pocas ayudas. En el último protocolo realizado duró más tiempo que en los anteriores.

Tabla 5.7. Ayudas utilizadas y tiempo de duración por el sujeto 7.

\begin{tabular}{|c||c|c||c||}
\hline $\begin{array}{c}\text { Nombre del } \\
\text { protocolo aplicado }\end{array}$ & $\begin{array}{c}\text { N. }{ }^{\circ} \text { del protocolo } \\
\text { aplicado }\end{array}$ & $\begin{array}{c}\text { N. }{ }^{\circ} \text { de ayudas } \\
\text { utilizadas }\end{array}$ & $\begin{array}{c}\text { Tiempo de } \\
\text { duración }\end{array}$ \\
\hline \hline muñecas & 4 & 3 & $12 \mathrm{~min}$ \\
\hline \hline muñecas & 5 & 2 & $10 \mathrm{~min} 17 \mathrm{seg}$ \\
\hline \hline muñecas & 6 & 4 & $14 \mathrm{~min}, 37 \mathrm{seg}$ \\
\hline
\end{tabular}


En la tabla 5.7., se observa que el sujeto 7 realizó tres protocolos de un mismo material. Se puede apreciar que la diferencia entre el número de ayudas es dispar. Es decir, del primero al segundo disminuyó, pero del segundo al tercero utilizó más ayudas que en los anteriores. El tiempo estuvo entre 10 y 15 minutos.

De estos resultados, se puede deducir que aunque algunos sujetos utilizaron mayor números de ayudas que otros, todos descubrieron la palabra oculta, lo cual indica que son niños que están en capacidad de construir conceptos artificiales para la vida cotidiana cuando se les brinda una ZDP por medio de un andamiaje (Wood \& Wood, 1996).

\subsection{Resultados del post-test}

Las pruebas de post-test se aplicaron a los niños después de que ellos realizaran los protocolos para descubrir la palabra oculta. Éstas consistieron en pedirles que expresaran, gráfica unos y verbalmente otros, qué significaba la palabra oculta.

Cuando los niños realizaron una representación gráfica, la mayoría no recordó la palabra oculta, sino que pudo reconocer el atributo que la caracterizaba.

En el anexo 3 se pueden apreciar los dibujos que realizaron cuatro niños. En éstos, se observa cómo los niños de 8 años son más detallistas en el momento de dibujar. Un ejemplo de esto fue un niño que dibujó completamente los carros, les colocó llantas, adhesivos y describió el color que caracterizaba cada carro (ver figura 6 en la siguiente página).

Por su parte, una niña de 6 años dibujó sólo el atributo de la palabra oculta, es decir, una cartera, pero no hizo la muñeca completa portando la cartera (ver figura 7 en la siguiente página). 


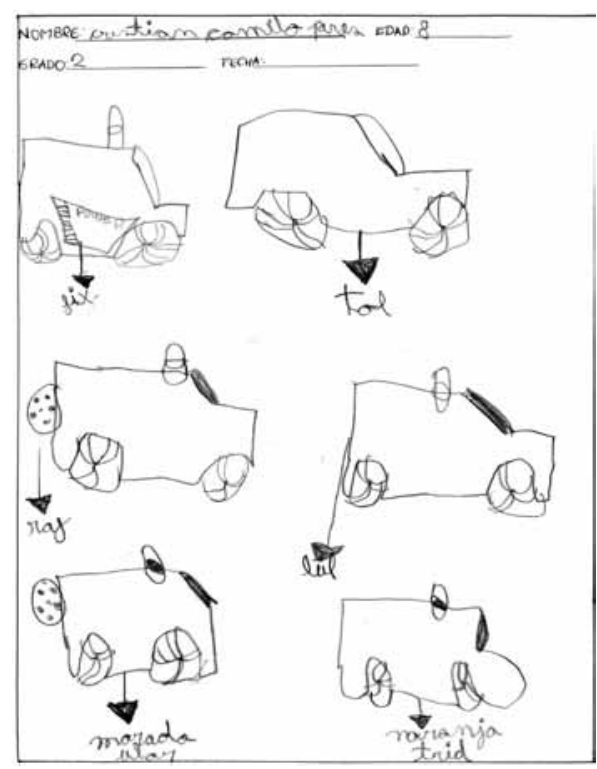

Figura 6. Resultado de post-test.

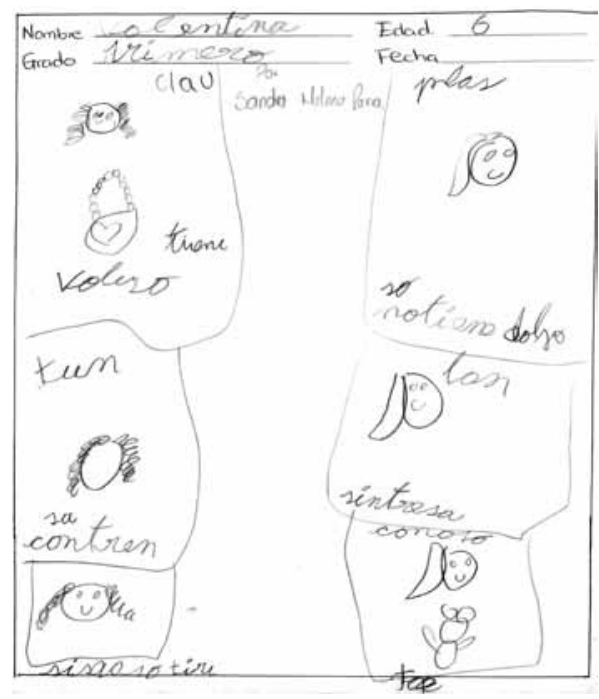

Figura 7. Resultado de post-test. 
En otro ejemplo, un niño también de 6 años, dibujó las casas, pero no las detalló muy bien (ver figura 8).

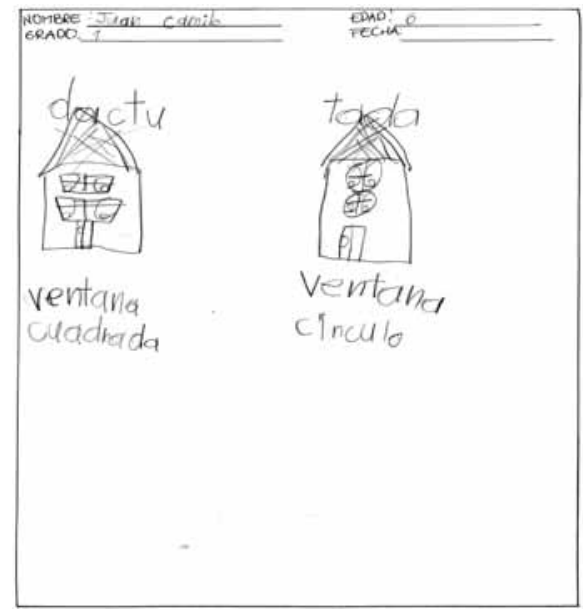

Figura 8. Resultado de post-test.

De lo anterior, es posible deducir que los niños de 8 años fijan más su atención en todos los detalles, y que esto hace que se les facilite descubrir la palabra oculta en comparación con los niños de 6 años, y así forman con más facilidad conceptos artificiales de la vida cotidiana.

\section{Conclusiones y discusión}

El propósito de esta investigación fue observar la formación de conceptos de la vida cotidiana en niños de 6 a 8 años de edad como manipulativos físicos para el aprendizaje. Para cumplir este objetivo, se diseñó un material (casas, muñecas, carros) con el cual se adaptó el experimento clásico método de la doble estimulación (Vigotsky 1995).

A diferencia de la prueba original, en la cual Vigotsky realiza la clasificación de los materiales por dos atributos, el realizado en 
esta investigación solamente presentó la clasificación por un solo un atributo.

Se hizo necesario elaborar protocolos de instrucción para llevar a cabo la aplicación de la prueba de una manera correcta. Este instrumento describió paso a paso el orden en el cual se debería desarrollar la prueba para poder ofrecer niveles de ayuda necesarios a los sujetos que participaron en ella.

De igual manera que lo hizo Vigotsky, se observó el proceso de formación conceptual de los niños. Éstos partieron del nivel de pensamiento en complejos, ya que los sujetos realizaron vínculos coherentes y verdaderos que existían entre el material diseñado y los agrupaban en familias separadas pero relacionadas. Finalmente, los sujetos llegaron a adquirir el último nivel de formación de conceptos, ya que lograron agrupar en un mismo conjunto los objetos que tenían mayor similitud, siendo todos los objetos distintos y agrupándolos teniendo en cuenta un atributo en común, llegando así a formar conceptos potenciales.

Durante el análisis de resultados de la prueba piloto se observaron claramente ciertas generalidades. Entre ellas se consideró que el desarrollo de la formación de conceptos en los niños no es homogénea, ya que se presentó el caso particular de un sujeto de 6 años que realizó la prueba con mayor facilidad que un sujeto de 8 años de edad. Se logra evidenciar que los sujetos de 8 años de edad son más minuciosos y observadores, lo que ocasiona que tomen más tiempo para clasificar los objetos por un atributo en común.

Adicionalmente, se pudo observar que la mayoría de participantes en la prueba piloto presentó dificultad al momento de expresar verbalmente la palabra oculta a la cual pertenecían los objetos con los que se llevó a cabo la prueba. Por otra parte, a todos los sujetos no se les facilitó expresar gráficamente los atributos por los cuales se clasificaban los objetos. Por tal razón, esto da a entender que a 
algunos sujetos se les facilitó expresar el conocimiento adquirido de manera verbal y a otros se les facilitó expresarlo por medio de gráficos.

Para finalizar esta discusión es conveniente decir que los manipulativos físicos son esenciales en las edades entre los 6 y 8 años de edad, ya que brindan al niño la oportunidad de tocar, coger, mover, trasladar y organizar su material de aprendizaje. De esta manera, se le facilita observar las características de los objetos y los detalles de los mismos, con el fin de formar un concepto potencial.

Por último, pero no menos importante, el presente estudio aportó el diseño y prueba piloto de manipulativos físicos para observar la formación de conceptos artificiales en niños de 6 a 8 años de edad en el contexto de la investigación más general, la cual busca estudiar los procesos de aprendizaje por medio de manipulativos que pueden presentarse en tres diferentes sustratos, a saber, físico, digital-mouse y digital-pantalla táctil.

\section{Bibliografía}

AINSWORTH, S. E. (2006). "DeFT: A conceptual framework for considering learning with multiple representations". En: Learning and Instruction, 16 (3), 183-198.

ARTINO, A. (2008). "Cognitive load theory and the role of learner experience: An abbreviated review for educational practitioners". En: AACE Journal , 16 (4), 425-439.

HERNÁNDEZ, R., FERNÁNDEZ, C., \& BAPTISTA, P. (2008). Metodología de la investigación. México: Mac Graw Hill.

LURIA, A. (1995). Conciencia y Lenguaje. Madrid: Editorial Visor.

MANCHES, A., \& O'MALLEY, C. (en prensa). Tangibles for learning: a representational analysis of physical manipulation. 1-24. 
MARTIN, T., \& SCHWARTZ, D. L. (2005). "Physically Distributed Learning: Adapting and Reinterpreting Physical Environments in the Development of Fraction Concepts". En: Cognitive Science , 29, 587-625.

NÚÑEZ, R., EDWARDS, L. D., \& MATOS, J. F. (1999). “Embodied Cognition as Grounding for Situatedness and Context in Mathematics Education". En: Educational Studies in Mathematics, 39 (1999), 45-65.

ORGILL, M., \& CRIPPEN, K. (2010). “Teaching with External Representations: The Case of a Common Energy-Level Diagram in Chemistry". En: Journal of College Science Teaching, 40 (1), 78-84.

VYGOTSKY, L. (1995). Pensamiento y lenguaje. Buenos Aires: Ediciones Fausto.

WOOD, D., \& WOOD, H. (1996). "Vygotsky, Tutoring and Learning". En: Oxford Review of Education, Vol. 22 (Número 1), pp. 5-12. 


\section{ANEXOS}

\section{Anexo I}

\section{Matriz de Casas}

\begin{tabular}{||c||c||c||}
\hline & & \\
\hline
\end{tabular}




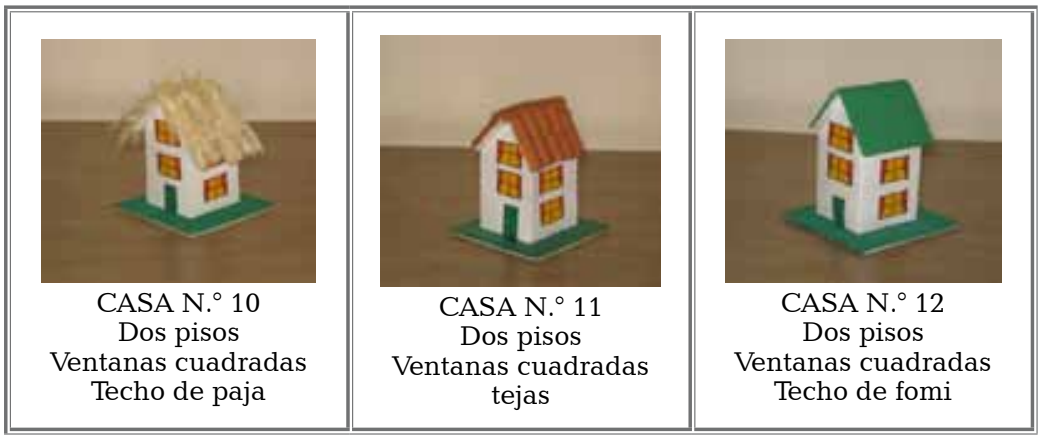

\section{Matriz de Muñecas}

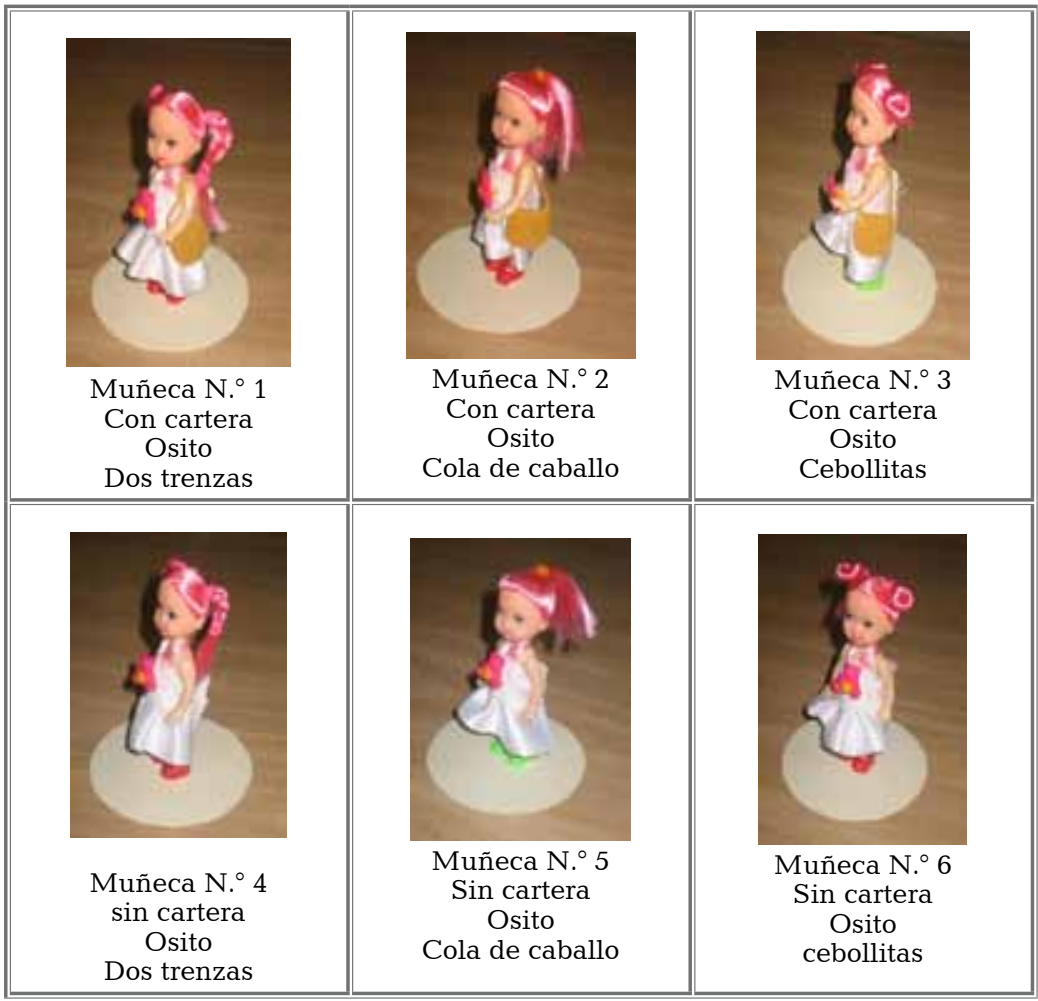




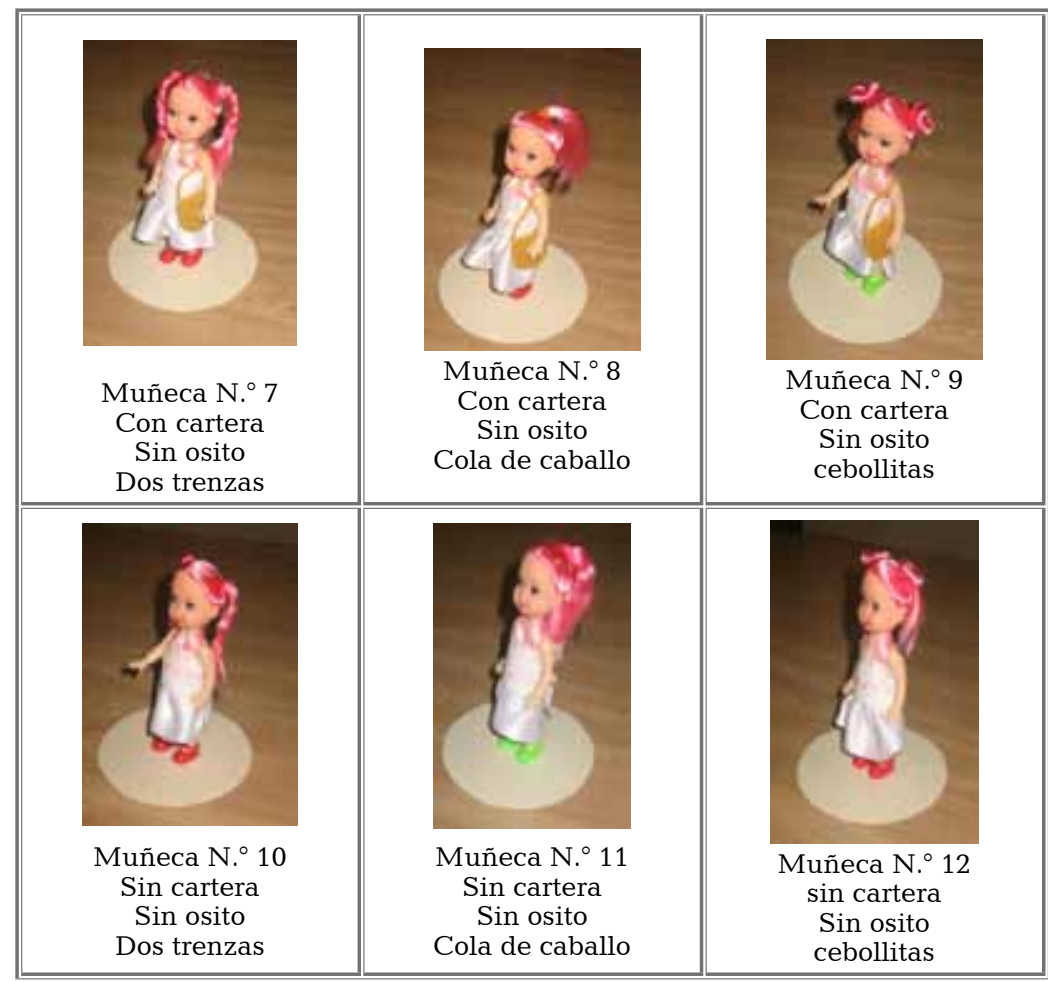

\section{Matriz de Carros}

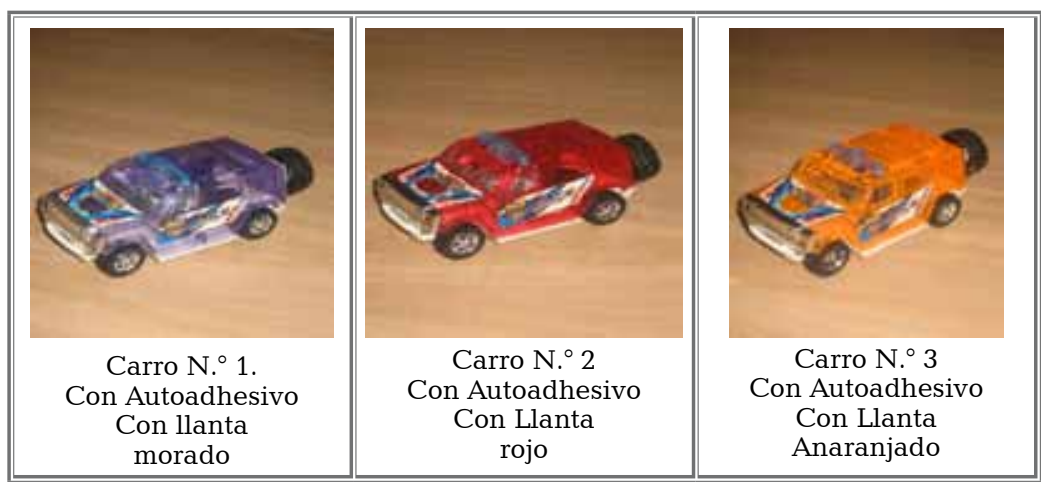




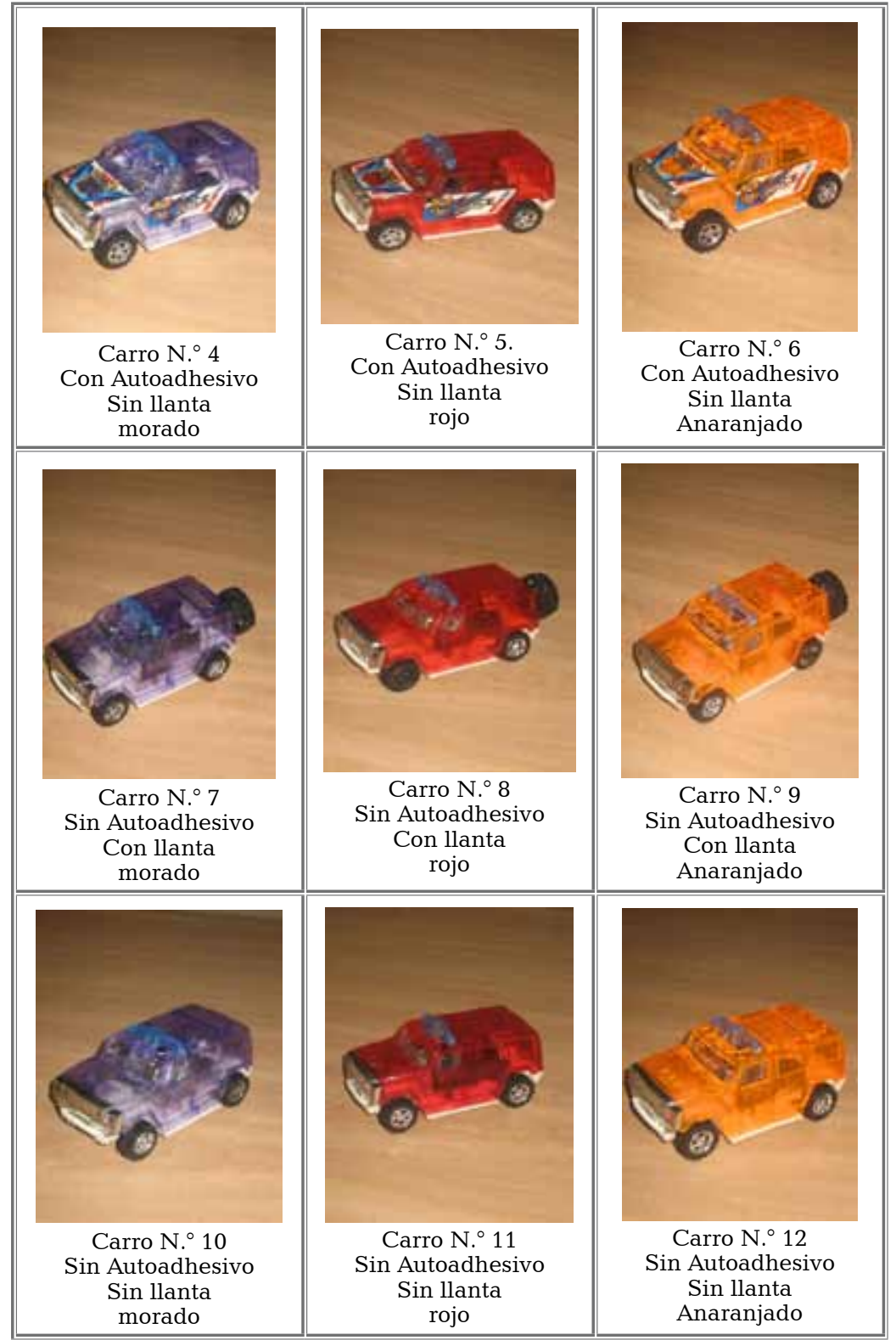

ITINERARIO EDUCATIVO • AÑO XXV, N. ${ }^{\circ} 57 \cdot 157-I 83 \cdot$ ENERO-JUNIO DE ZOII 


\section{Anexo 2}

Tarjetas de la prueba "Cuarto Excluido"

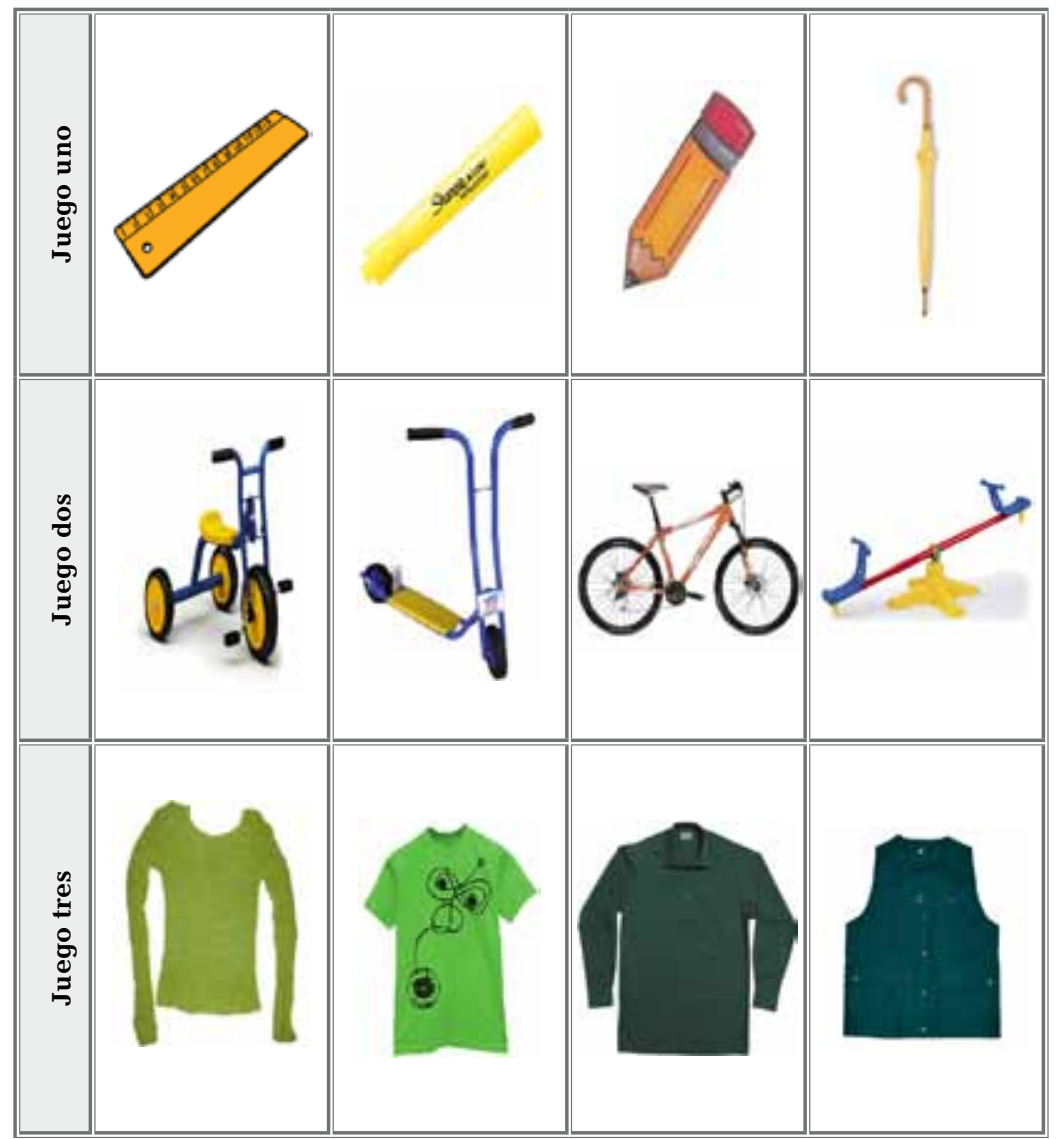

Tarjetas prueba del cuarto excluido. En el juego numero uno la categoría es útiles escolares; en el segundo juego la categoría es juguetes con ruedas; y en el tercer juego la categoría es pendas de vestir con mangas. 


\section{Anexo 3}

\section{Prueba Post-test}
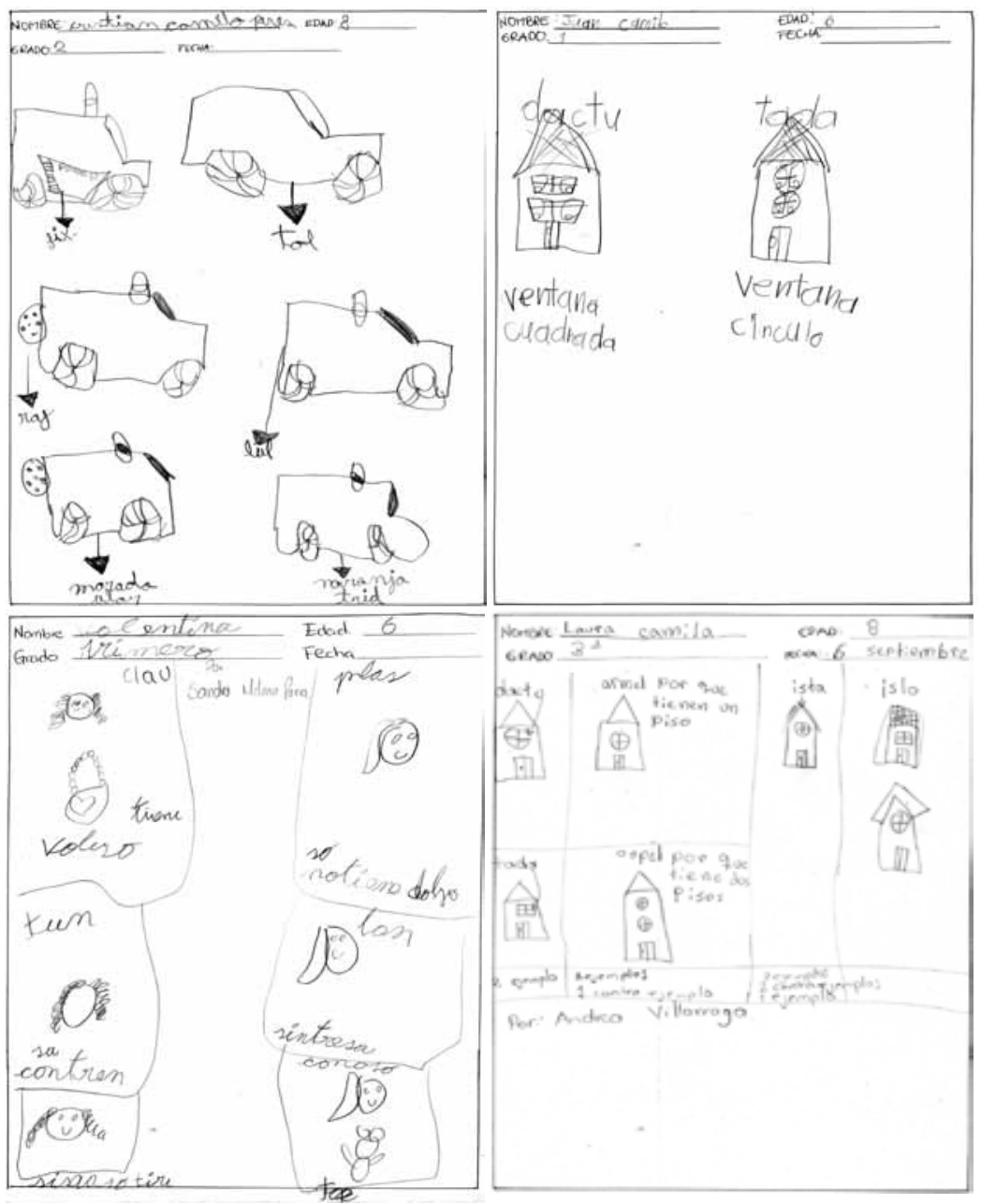

Recibido $\in$ n octubre 2010 Arbitrado en diciembre 2010

ITINERARIO EDUCATIVO • AÑO XXV, N. ${ }^{\circ} 57 \cdot 157-I 83 \cdot$ ENERO-JUNIO DE ZOII 